\title{
Educação em saúde através dos meios de comunicação: contribuindo para a formação dos estudantes de enfermagem*
}

\author{
Health education through means of communication: contributing to the formation \\ of nursing students
}

\section{Educación en salud a través de los medios de comunicación: contribuyendo para la formación de los estudiantes de enfermería}

\author{
Recebido: 18/02/2018 \\ Aprovado: 20/09/2018 \\ Publicado: 29/01/2019
}

\author{
Isabela Aparecida Gonçalves Prada ${ }^{1}$ \\ Sabrina Karla de Souza Cubas ${ }^{2}$ \\ Divanice Contim ${ }^{3}$ \\ Fernanda Carolina Camargo ${ }^{4}$
}

Este estudo tem como objetivo relatar uma experiência sobre a utilização de mídias (rádio e jornal) para difusão e acesso a informações seguras sobre cuidado em saúde, enquanto estratégia extracurricular na formação de enfermeiros. Teve como arcabouço conceitual metodológico a Literacia em Saúde, processo constituído por: compreensão e avaliação crítica das informações a serem difundidas e empreendimento de informações seguras sobre saúde capazes de apoiar a tomada de decisão. Iniciou-se em 2013 utilizando-se de veículos comunicacionais locais e universitários. Publicações dos textos informativos e dos assuntos reproduzidos na rádio foram definidas pelos acadêmicos. De forma geral, a difusão de informações seguras em saúde tem sido considerada prática potencial à transformação das condições de vida das populações. A utilização de mídias locais contextualiza a linguagem ao público alvo, permitindo maior amplitude de acesso das populações às informações. Sendo esta uma atividade extracurricular, possibilitou-se aos graduandos vivenciarem espaços de tecnologias inovadoras para a promoção da saúde.

Descritores: Educação em saúde; Meios de comunicação; Estudantes de enfermagem.

This study is an experience report on the use of certain media (radio and newspapers) for the dissemination and access to safe information about healthcare, as an extracurricular strategy in the formation of nurses. Its conceptual methodological framework was Health Literacy, which is a process made up of: understanding and critical evaluation of the information to be disseminated, the use of safe information regarding healthcare that can aid in decision-making. It started in 2013, using local and university means of communication. The undergraduate students decided about the publishing of informative texts and the themes to be transmitted through the radio. In general, the dissemination of safe information on health has been considered as a practice with the potential of transforming the life conditions of the populations. The use of local media contextualizes the language to the target audience, allowing for a broader access to the information by the population. Since this is an extracurricular activity, undergraduates could experience spaces of innovative technologies for health promotion.

Descriptors: Health education; Communications media; Students nursing.

Este estudio tiene como objetivo relatar una experiencia sobre la utilización de medios de comunicación (radio y periódico) para difusión y acceso a informaciones seguras sobre cuidado en salud, como estrategia extracurricular en la formación de enfermeros. Tuvo como arcabuz conceptual metodológico la Alfabetización en Salud. Proceso constituido por: comprensión y evaluación crítica de las informaciones a ser difundidas, emprendimiento de informaciones seguras sobre salud capaces de apoyar la toma de decisiones. Se inició en 2013 utilizando vehículos comunicativos locales y universitarios. Publicaciones de los textos informativos y de los asuntos reproducidos en la radio fueron definidos por los académicos. De forma general, la difusión de informaciones seguras en salud ha sido considerada como práctica potencial para la transformación de las condiciones de vida de las poblaciones. La utilización de medios de comunicación locales, contextualiza el lenguaje al público-objetivo, permitiendo mayor amplitud de acceso de las poblaciones a las informaciones. Como siendo actividad extracurricular posibilitó a los graduandos vivenciar espacios de tecnologías innovadoras para la promoción de la salud.

Descriptores: Educación en salud; Medios de comunicación; Estudiantes de enfermería.

1. Acadêmica de Enfermagem da Universidade Federal do Triângulo Mineiro (UFTM). Uberaba, MG, Brasil. ORCID: 0000-0001-8930-9239 E-mail: isaprada@outlook.com

2. Acadêmica em Enfermagem da UFTM, Uberaba, MG, Brasil. ORCID: 0000-0002-3112-9515. E-mail: sasa.souza1501@hotmail.com

3. Enfermeira. Mestre em Enfermagem. Doutora em Ciências. Professora Adjunta da UFTM, Uberaba, MG, Brasil. ORCID: 0000-0001-5213-1465 E-mail: d.contim@uol.com.br

4. Enfermeira. Mestre e Doutora em Atenção à Saúde. Epidemiologista Clínica da Gerência de Ensino e Pesquisa do Hospital de Clínicas (GEP-HC) da UFTM, Uberaba, MG, Brasil. ORCID:0000-0002-1048-960X E-mail: fernandaccamargo@yahoo.com.br

*Fonte de Financiamento: Secretaria de Educação Superior do Ministério da Educação/SESU-MEC. 


\section{INTRODUÇÃO}

D iariamente, por meio da internet, são publicadas milhares de notícias sobre diversos temas, como: política, esporte, entretenimento, cultura, educação e saúde. Porém, mesmo sendo obtidas com facilidade de acesso, as informações nas mídias devem ser analisadas com cautela. É preciso analisar criticamente a sua veracidade antes mesmo de serem assumidas como condutas ${ }^{1}$.

Segundo a Secretaria de Comunicação Social da Presidência da República, na Pesquisa Brasileira de Mídia de 2015, realizada em 848 municípios com 18.312 entrevistados maiores de 16 anos, evidenciase que cerca de $55 \%$ dos entrevistados ouviram uma emissora de rádio uma vez por semana e $22 \%$ leem semanalmente. Destacase que rádio e jornal ainda se mantém como cruciais veículos de informações no Brasil, mesmo frente ao advento da internet ${ }^{2}$.

Nessa direção, compreende-se que as emissoras de rádio e o jornal são importantes veículos para se difundir a comunicação sobre condutas de bem-estar. Ressalta-se que informações em saúde são cruciais para conhecimento e adoção de comportamentos saudáveis. Entretanto, é preciso assegurar a qualidade das informações difundidas pelas mídias. Dessa maneira, a qualidade da informação em saúde relaciona-se a: seleção do conteúdo, seu potencial de aplicabilidade para resolução de problemas e orientação na tomada de decisões. Frente a esse processo, o conhecimento seguro contribui para a autonomia em saúde das pessoas ${ }^{3}$.

A autonomia é princípio da promoção da saúde, garantida nas metas do milênio da Organização das Nações Unidas para 2030, e se direciona à ampliação do acesso a informações seguras para o cuidado benéfico. Portanto, o escopo é a universalidade, expresso pela necessidade de se assegurar uma vida saudável e promover o bem-estar para todos, em todas as idades ${ }^{4}$. Nesse contexto, a enfermagem apresenta-se como protagonista mundial para o alcance de um nível melhor de saúde para os povos ${ }^{5}$.

Mas existe uma escassez de profissionais de enfermagem qualificados relacionada à má distribuição geográfica desses trabalhadores entre os países. Essa realidade se expressa frente a composição de habilidades e competências que permeiam a formação deste profissional ${ }^{5}$.

Questionamentos emergem sobre como o enfermeiro pode utilizar-se de mídias para instrumentalizar a população em seus cuidados à saúde, promovendo autonomia para o autocuidado. Em específico para o Brasil, onde a universalidade do acesso à saúde é garantida como direito constitucional, observa-se a necessidade do empreendimento de experiências que abordem a maneira de enfermeiros atuarem nas mídias, veiculando informações para a autonomia e a promoção da saúde 5 .

A utilização das mídias para a informação em saúde, apesar de ser cotidiana na contemporaneidade, uma vez que as pessoas se encontram conectadas em diferentes plataformas, ainda se apresenta como espaço de produção científica pouco explorado $^{6,7}$. Mediante a realidade explicitada, o presente estudo tem por objetivo relatar a experiência sobre a utilização de mídias - rádio e jornal - para a difusão e acesso a informações seguras sobre cuidado em saúde, enquanto estratégia extracurricular na formação de enfermeiros.

\section{MÉTODO}

Trata-se de um relato de experiência sobre a inciativa de graduandos de enfermagem participantes de um Programa de Educação Tutorial (PET), quanto à utilização de mídias informacionais para difusão de práticas em saúde.

Criado em 2006, no eixo base das Políticas Públicas de Saúde, o PET Enfermagem da Universidade Federal do Triângulo Mineiro (UFTM) agrega, sob a orientação de um professor tutor, alunos bolsistas e voluntários que desenvolvem atividades com vistas a garantir aos alunos experiências não presentes na grade curricular convencional, visando à formação acadêmica global ${ }^{8}$.

A orientação da experiência ancorou-se tanto na sua descrição como no desenvolvimento, na perspectiva do 
arcabouço teórico metodológico do "Letramento em Saúde". Esta teoria pode ser definida como: conjunto de habilidades que envolvem o conhecimento, a motivação, e as competências para acessar, compreender, avaliar, e aplicar informações de saúde, para julgamentos e tomada de decisões cotidianas sobre os cuidados de saúde, prevenção de doenças e promoção da saúde para manter ou melhorar a qualidade de vida durante o curso da vida9.

Literacia em Saúde é um termo que se iniciou na década de 1970 nos Estados Unidos, período marcado pela luta dos ativistas dos movimentos sociais para garantia da justiça social, onde pode ser observada uma crescente demanda por uma linguagem mais clara nas mensagens produzidas no âmbito de programas de saúde pública nos Estados Unidos ${ }^{10}$.

No Brasil, este termo foi traduzido em seu sentido denotativo: Letramento. Entretanto essa temática ainda é pouco difundida. Ela ainda afeta o acesso e a compreensão de informações da população sobre os cuidados com a saúde e prevenção de doenças. Por outro lado, o baixo letramento é frequentemente acompanhado por vergonha e menor capacidade de entender como prevenir doenças e promover saúde ${ }^{11}$.

0 alcance desta teoria integra um processo constituído por: 1) acesso ao conhecimento; 2) compreender a informação; 3) avaliar os dados seguros sobre saúde; 4) exercer o julgamento crítico; 5) analisar e tomar decisões com base nas referências sobre saúde; e 6) resolver problemas.

Frente a essas perspectivas, a tese citada apresenta-se como fundamental para o desenvolvimento social, econômico e da saúde, sendo a educação um processo vital para que isso ocorra. Dessa forma, resulta-se em habilidades para manutenção do autocuidado e participação na tomada de decisões ${ }^{12-14}$.

Considerando esses aspectos, a experiência a ser relatada utilizou para o fortalecimento da Literacia em Saúde o provimento de informações seguras a partir das mídias locais, Rádio Universitária e Jornal Abadia Noticia, ambos da cidade de UberabaMG, obedecendo o fluxo apresentado na Figura 1.

Figura 1. Fluxograma para o desenvolvimento da atividade Educação em Saúde nas Mídias do grupo PET Enfermagem. Uberaba, Minas Gerais, 2017.

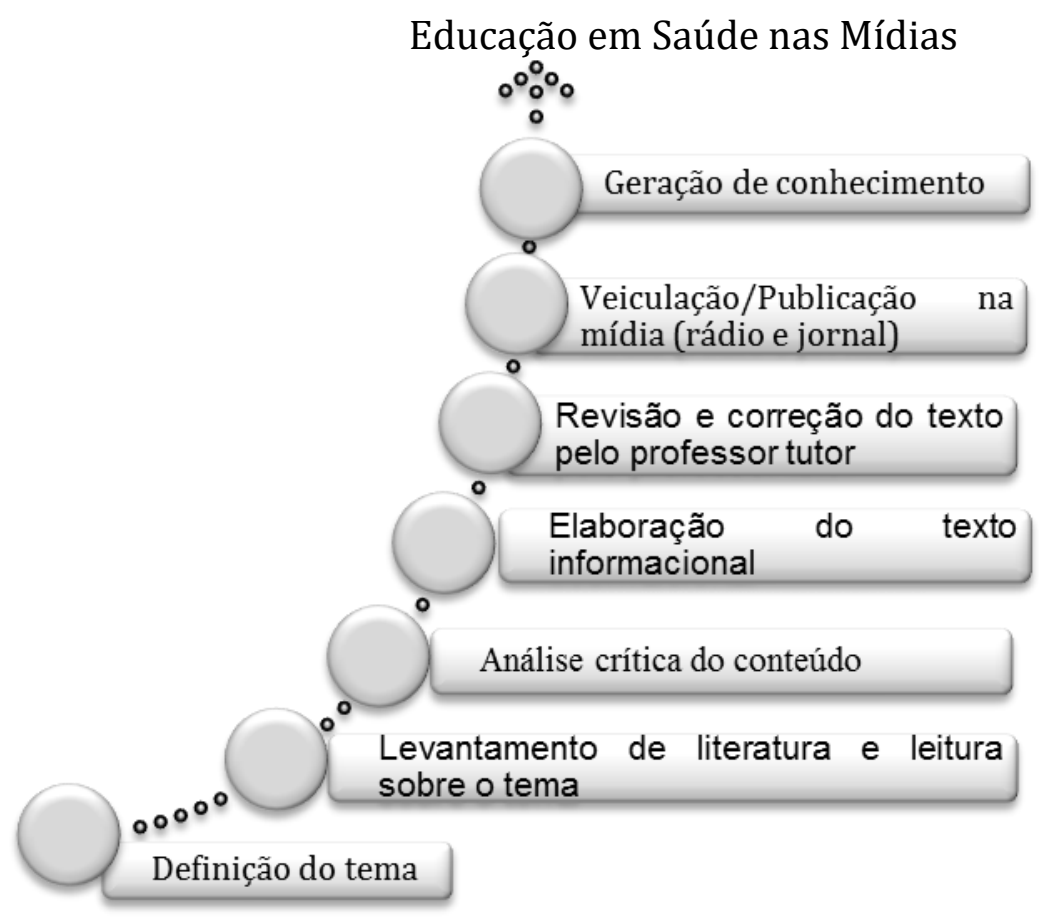




\section{RESULTADOS}

A atividade de Educação em Saúde nas mídias é composta por quatorze discentes e um docente tutor, todos membros do Programa de Educação Tutorial-PET (Figura 1). A atividade de Educação em Saúde nas Mídias, na mídia Rádio Universitária 104.9 Mhz, se iniciou no ano de 2015, já na mídia Jornal Abadia Notícia, se iniciou no ano de 2013.

São aproximadamente quatro anos de intenso trabalho e dedicação, sob a orientação do professor tutor e colaboração de profissionais convidados especialistas que auxiliam na elaboração dos textos informativos sobre o tema de saúde a ser publicação no jornal ou veiculado na rádio.

A mídia Rádio Universitária 104.9 Mhz atinge o público das classes " $A$ " e " $B$ ", tendo penetração nas demais classes pela programação versátil e qualificada. Possui abrangência regional, incluindo toda região do Triângulo e do Alto Paranaíba do Estado de Minas Gerais, no raio de alcance de aproximadamente $180 \mathrm{~km}$ de Uberaba-MG. Alcança cidades como Conceição das Alagoas/MG, Campo Florido/MG, Delta/MG, Ponte Alta/MG, Planura, Veríssimo/MG, Conquista/MG, Sacramento/MG, Nova Ponte/MG, Santa Rosa/MG, Santa Juliana/MG, Almeida Campos/MG, Frutal/MG, Água Comprida/MG, Aramina/SP, Igarapava/SP, Ituverava/SP, Pedregulho/SP, Franca/SP entre outras.

A mídia Jornal Abadia Notícia, de veiculação gratuita, tem duas formas de divulgação, a forma impressa, com distribuição de 50 mil exemplares mensais com abrangência local destinada ao município de Uberaba-MG, e a forma virtual, disponível em sítio eletrônico (internet) com potencial para atingir um público ainda maior.

Os temas da atividade de Educação em Saúde na mídia Rádio Universitária 104.9 Mhz de Uberaba, têm sido definidos a partir do calendário temático de saúde do Ministério da Saúde e/ou pela direção da Rádio.

A partir da definição do tema, sob a orientação/supervisão do professor tutor, o aluno faz um levantamento das melhores evidências sobre o tema, elabora a minuta do texto e encaminha para análise e correções do professor tutor e nos casos necessários é encaminhado para algum colaborador especialista no assunto, geralmente professor da Universidade ou Profissional de saúde do Hospital de Clínicas da UFTM.

Após revisão e adequação da linguagem pelo jornalista da rádio, os textos, com duração de até um minuto, foram gravados no estúdio da Rádio Universitária ou pelo próprio aluno em telefone celular. Quando a gravação foi feita no celular do aluno, foi encaminhada ao profissional responsável da Rádio para o tratamento técnico, e em alguns casos este procedimento foi repetido. Cada texto foi veiculado três vezes ao dia em horário definido pela direção técnica da Rádio durante uma ou duas semanas. A partir do ano de 2018 os textos foram veiculados durante todo o mês corrente.

Quanto à mídia Jornal Abadia Notícia, os temas têm sido definidos ou sugeridos mensalmente pelo editorial do jornal, geralmente temas de grande relevância e interesse da população. Alguns temas foram propostos pela equipe do Pet-Enfermagem.

Similar à mídia Rádio Universitária, a partir da definição do tema, uma dupla ou um trio de alunos, sob a orientação/supervisão do professor tutor, faz um levantamento das melhores evidências sobre o tema, elabora a minuta do texto, e a encaminha para análise e correções do professor tutor ou de algum outro profissional experiente no assunto. Este texto tem sido enviado, até o $15^{\circ}$ dia do mês, à Editora do Jornal para tratamento técnico e veiculação na Coluna Mais Saúde do periódico.

Alguns dos temas veiculados na Rádio Universitária e/ou publicados no Jornal Abadia Notícia no ano de 2017 foram: 1) HPV - Vírus do papiloma humano, 2) Febre Amarela, 3) Tuberculose, 4) Violência Sexual, 5) Maio Amarelo, 6) Doação de Sangue, 7) Teste do Pezinho, e 8) Doenças Comuns no Inverno. Já os assuntos abordados na rádio foram: 1) Febre Amarela, 2) Deficiência Intelectual/Síndrome de Down, 3) HIV -Vírus da imunodeficiência humana, 4) Saúde do Trabalhador, 5) Doenças Renais, 6) Hábitos 
Saudáveis, 7) Hipertensão, 8) Doença de Parkinson, 9) Câncer Infanto-juvenil, 10) Tabagismo, 11) Infecções Urinárias, 12) Glaucoma, 13) Teste do Pezinho, 14) Hepatites Virais, 15) Alzheimer, 16) Colesterol, 17) Doação de Sangue, 18) Diabetes, e 19) Dia Nacional do Trânsito.

\section{DISCUSSÃO}

0 ato de se comunicar percorre todo o histórico da humanidade, e está interligado a diversas áreas do conhecimento, a exemplo da saúde, como forma de garantir que este direito seja atendido e alcance todos os cidadãos ${ }^{15}$.

No setor da saúde, assim como nos demais setores da sociedade, o uso das novas tecnologias se faz necessário para ampliar o conhecimento e empoderar a comunidade com informações. Nesse contexto, um desafio emergente é o de ampliar o acesso da população às informações seguras sobre saúde e autocuidado ${ }^{16}$.

Com isso, a difusão de informações se mostra uma prática potencial para transformação das condições de saúde e vida das populações. Por conseguinte, as mídias informacionais se apresentam como ferramentas cruciais para o trabalho de profissionais de saúde, quando se pretende disseminar informações sobre doenças, promoção e prevenção de agravos ${ }^{17}$.

A própria população tende a utilizar as mídias informacionais como um espaço para expor suas experiências sobre o adoecimento, trocar ideias, manifestar angústias, proporcionando a esse espaço virtual formas colaborativas, interativas e até mesmo terapêuticas para resolução de suas demandas em saúde ${ }^{17}$.

As informações sobre saúde, veiculadas pelas mídias, tendem a ampliar o empoderamento das populações em seus cuidados à saúde. Antes de tudo, utilizar as mídias informacionais para difusão de práticas seguras de saúde coaduna-se sobremaneira com o Letramento em saúde, sendo a principal consequência dessa prática informacional ampliar críticas na absorção de conteúdos pertinentes para escolha de como levar uma vida saudável ${ }^{18}$.
No entanto, embora as mídias sejam ferramentas potencializadoras de saúde, a inserção da Literacia em saúde ainda é um constructo. Isso pode ser observado através de pesquisas em bases de dados, as quais indicam um pequeno número de publicações a respeito do tema no Brasil ${ }^{17}$. Entretanto, a utilização de mídias pode interferir positivamente no processo de aprendizagem e crítica sobre melhores práticas de saúde, levando à autonomia dos sujeitos envolvidos para a condução do seu devir'18.

Apesar deste relato tratar-se de uma experiência local sobre a utilização de mídias informacionais, considera-se que esta experiência possa ser replicada em cenários similares, em especial para contextos que envolvam a integração na formação de experiências extramuros para a promoção da saúde.

0 contato de acadêmicos de enfermagem com novas tecnologias como as mídias informacionais expande potencialmente os horizontes de formação desses profissionais, que passam a compreender modos de cuidar em saúde, frente a difusão de informações que alcancem populações em diferentes localidades. Esta experiência ainda é potencial para discussão de iniciativas empreendidas em programas de educação tutorial, tendo em vista as escassas publicações sobre o tema.

\section{CONCLUSÃO}

A disseminação de informações sobre saúde pelas mídias informacionais tem potencial para contribuir na criação de competências interacionais para julgamento crítico, autonomia, e decisões em saúde a partir de informações seguras.

A utilização de mídias locais como Rádio e Jornal, de forma geral, contextualiza a linguagem ao público alvo, permitindo uma maior amplitude de acesso das populações a informações seguras sobre práticas de saúde.

Operacionalmente, o desenvolvimento desta atividade em âmbito acadêmico, como uma atividade extracurricular possibilita capacitar os profissionais de saúde, sobretudo enfermeiros, a vislumbrarem 
espaços e tecnologias inovadoras para a promoção da saúde.

Em muito, o contato com esta experiência ainda na graduação viabiliza aos profissionais de saúde empreenderem estratégias comunicacionais, considerando a disseminação de cuidados de saúde propriamente ditos; orientações para a prevenção de doenças, e divulgação de ações de promoção de saúde.

Com isso, pesquisas futuras são sugeridas para avaliação do impacto dessa iniciativa, tanto da audiência (pessoas que utilizam as informações) como dos próprios acadêmicos, sobre como essa intervenção vem modificando a formação no ensino superior para que, cada vez mais, esteja aproximada às necessidades reais de saúde das populações.

\section{REFERÊNCIAS}

1. Medeiros AS, Magalhães R, Pereira JR. Lei de acesso à informação: em busca da transparência e do combate à corrupção. Inf Inf. [Internet]. 2014 [citado em 27 ago 2017]; 19(1):55-75.

\subsection{4v19n1p55}

2. Presidência da República (Brasil), Secretaria de Comunicação Social. Pesquisa brasileira de mídia 2015: hábitos de consumo de mídia pela população brasileira. Brasília, DF: SECOM; 2014, 156p.

3. Lisboa SSM. Jornalismo e a credibilidade percebida pelo leitor: independência, imparcialidade, honestidade, objetividade e coerência. [dissertação]. Porto Alegre: Universidade Federal do Rio Grande do Sul; 2012.112p.

4. Health in the post-2015 agenda: report of the global thematic consultation on health [Internet]. [S.l.: World Health Organization]; 2013 [citado em 27 ago 2017]. Disponível em:

https://www.paho.org/arg/images/gallery/ Health\%20post-2015\%20Apr13.pdf?ua=1

5. Mendes IAC, Ventura CAA. Protagonismo da enfermagem nas metas da ONU para a saúde dos povos. Rev Latinoam Enferm. [Internet]. 2017 [citado em 27 ago 2017]; 25:e2864. ttp://dx.doi.org/10.1590/1518-

8345.0000.2864

6. Bermejo L. Envejecimiento activo, pedagogia gerontológica y buenas prácticas socioeducativas con personas adultas mayores. Rev Interv Socioeducativa [Internet]. 2012 [citado em 27 ago 2017]; 51:27-44. Disponível em: http://www.raco.cat/index.php/EducacioSoc ial/article/view/260500/369075

7. Condeza AR, Bastías G, Valdivia G, Cheix C, Barrios X, Rojas R, et al. Adultos mayores en Chile: descripción de sus necessidades en comunicación en salud preventiva. Cuad Info. [Internet]. 2016 [citado em 27 ago 2017]; (38):85-104.

doi:

https://doi.org/10.7764/cdi.38.964

8. Ministério da Educação (Br). Secretaria de Educação Superior. Manual de Orientações básicas - PET. Brasília, DF: Ministério da Educação; 2006.

9. Sörensen K, Van den Broucke S, Fullam J, Doyle G, Pelikan J, Slonska Z, et al. Health literacy and public health: a systematic review and integration of definitions and models. BMC Public Health [Internet]. 2012 [citado em 27 ago 2017]; 12(1):80. doi: http//dx.doi.org/ 10.1186/1471-2458-12-80 10. Nutbeam D. The evolving concept of healt literacy. Soc Sci Med. [Internet]. 2008 [citado em jul 2017]; 67(12):2072-8. Disponível em: https://www.sciencedirect.com/science/arti cle/pii/S0277953608004577?via\%3Dihub

11. Quemelo PRV, Milani D, Bento VF, Vieira ER, Zaia JE. Literacia em saúde: tradução e validação de instrumento para pesquisa em promoção da saúde no Brasil. Cad Saúde Pública [Internet]. 2017 [citado em 27 ago 2017]; 33(2):e00179715. doi: http://dx.doi.org/10.1590/0102-

\section{1x00179715}

12. Nutbeam D. Health literacy as a public goal: a challenge for contemporary health education and communication strategies into the 21st century. Health Promot Int. [Internet]. 2000 [citado em 27 ago 2017]; 15(3):259-67. Disponível em: https://academic.oup.com/heapro/article/1 5/3/259/551108

13. Minghelli B. A importância da literacia para a saúde no controle do excesso de peso 
infanto-juvenil. RBONE. [Internet]. 2012 [citado em 27 ago 2017]; 32(6):96-105. Disponível http://www.rbone.com.br/index.php/rbone /article/view/193/255

14. Luís FSL. Literacia para a saúde e alimentação saudável: os novos produtos e a escolha dos alimentos. [tese]. Lisboa, Pt: Escola Nacional de Saúde Pública; 2010. 277p.

15. Almeida M. A promoção da saúde nas mídias sociais: uma análise do perfil do Ministério da Saúde no Twitter. [Trabalho de Conclusão de Curso]. Goiânia: Universidade Federal de Goiás; 2012.16p.

16. Pinto ACS, Scopacasa LF, Bezerra LLAL, Pedrosa JV, Pinheiro PNC. Uso de tecnologias da informação e comunicação na educação em saúde de adolescentes: revisão integrativa. Rev Enferm UFPE online. [Internet]. 2017 [citado em 27 ago 2017]; 11(2):634-44.

doi: https://doi.org/10.5205/1981-8963v11i2a11983p634-644-2017

17. Cruz DI, Daher RR, Dias PWS, Martins VF, Gandolfi PE. O uso das mídias digitais na educação em saúde. Cad FUCAMP. [Internet]. 2011 [citado em 22 set 2017]; 10(13):13042.

Disponível

em:

http://www.fucamp.edu.br/editora/index.ph p/cadernos/article/view/215/228.

18. Silva MJ, Linhares RN, Chagas AM, Santos M. Reflexões sobre a produção acadêmica em mídias, saúde e educação no Brasil. In: 4o Congresso Ibero-Americano em Investigação Qualitativa; 6o Simpósio Internacional de Educação e Comunicação; 5-7 ago 2015; Aracajú, SE. Linhares RN, Costa AP, editores. Atas do 6o Simeduc. [Internet]. [S.l.]: Ludomedia; 2015 [citado em 12 set 2017]. v. 5, p. 99-104. Disponível em: http://www.proceedings.ciaiq.org/index.php /ciaiq2015/article/view/202

\section{CONTRIBUIÇÕES}

Isabela Aparecida Gonçalves Prada, Sabrina Karla de Souza Cubas e, Fernanda Carolina Camargo participaram da concepção, busca bibliográfica, e redação. Divanice Contim e Fernanda Carolina Camargo contribuíram na redação e revisão crítica.

\footnotetext{
Como citar este artigo (Vancouver)

Prada IAG, Cubas SKS, Contim D, Camargo FC. Educação em Saúde através dos meios de comunicação: contribuindo para formação dos estudantes de Enfermagem. REFACS [Internet]. 2019 [citado em inserir dia, mês e ano de acesso]; 7(1):95-101. Disponível em: inserir link de acesso. DOI: inserir link do DOI.

Como citar este artigo (ABNT)

PRADA, I. A. G. et al. Educação em Saúde através dos meios de comunicação: contribuindo para formação dos estudantes de Enfermagem. REFACS, Uberaba, MG, v. 7, n. 1, p. 95-101 2019. Disponível em: <inserir link de acesso>. Acesso em: inserir dia, mês e ano de acesso. DOI: inserir link do DOI.

\section{Como citar este artigo (APA)}

Prada, I. A. G.; Cubas, S. K. S.; Contim, D. \& Camargo, F. C. (2019). Educação em Saúde através dos meios de comunicação: contribuindo para formação dos estudantes de Enfermagem. REFACS, 7(1), 95-101. Recuperado em: inserir dia, mês e ano de acesso de inserir link de acesso. DOI: inserir link do DOI
} 